\title{
Involvement of PI3K/AKT and MAPK Pathways for TNF-a Production in SiHa Cervical Mucosal Epithelial Cells Infected with Trichomonas vaginalis
}

\author{
Jung-Bo Yang ${ }^{1, \dagger}$, Juan-Hua Quann ${ }^{2, \dagger}$, Ye-Eun Kim ${ }^{3, \dagger}$, Yun-Ee Rhee', Byung-Hyun Kang', In-Wook Choi', \\ Guang-Ho Cha ${ }^{4}$, Jae-Min Yuk ${ }^{4}$, Young-Ha Lee ${ }^{4, *}$ \\ ${ }^{1}$ Department of Obstetrics and Gynecology, Chungnam National University School of Medicine, Daejeon 301-131, Korea; ${ }^{2}$ Department of \\ Gastroenterology, The Affiliated Hospital of Guangdong Medical College, Zhanjiang 524-001, Guangdong, China; ${ }^{3}$ Department of Biomedical \\ Science, Chungnam National University School of Medicine, Daejeon 301-131, Korea; ${ }^{4}$ Department of Infection Biology, Chungnam National \\ University School of Medicine, Daejeon 301-131, Korea
}

\begin{abstract}
Trichomonas vaginalis induces proinflammation in cervicovaginal mucosal epithelium. To investigate the signaling pathways in TNF-a production in cervical mucosal epithelium after T. vaginalis infection, the phosphorylation of PI3K AKT and MAPK pathways were evaluated in T. vaginalis-infected SiHa cells in the presence and absence of specific inhibitors. T. vaginalis increased TNF-a production in SiHa cells, in a parasite burden-dependent and incubation time-dependent manner. In T. vaginalis-infected SiHa cells, AKT, ERK1/2, p38 MAPK, and JNK were phosphorylated from $1 \mathrm{hr}$ after infection; however, the phosphorylation patterns were different from each other. After pretreatment with inhibitors of the PI3K/AKT and MAPK pathways, TNF-a production was significantly decreased compared to the control; however, TNF-a reduction patterns were different depending on the type of PI3K/MAPK inhibitors. TNF-a production was reduced in a dose-dependent manner by treatment with wortmannin and PD98059, whereas it was increased by SP600125. These data suggested that PI3K/AKT and MAPK signaling pathways are important in regulation of TNF-a production in cervical mucosal epithelial SiHa cells. However, activation patterns of each pathway were different from the types of PI3K MAPK pathways.
\end{abstract}

Key words: Trichomonas vaginalis, SiHa cell, PI3K/AKT, MAPK, TNF-a

\section{INTRODUCTION}

Trichomonas vaginalis is a flagellated protozoan parasite that infects the female reproductive tract and male urethra. It is one of the most common causes of non-viral sexually transmitted diseases in the world. Each year, about 160-180 million people are infected with T. vaginalis worldwide [1-3]. Infection with this parasite leads to vaginitis with burning, itching, and inflammation of the vaginal epithelium. Associated morbidities include endometritis, preterm birth, and other viral infections $[1,2]$. In addition, T. vaginalis is linked to cervical cancer, inflammatory diseases, and infertility [4]. Epidemiological studies have also reported that T. vaginalis can act as a cofactor

- Received 8 July 2015, revised 29 July 2015, accepted 30 July 2015.

*Corresponding author (yhalee@cnu.ac.kr)

†Jung-Bo Yang, Juan Hua Quan, and Ye-Eun Kim contributed equally to this work.

(c) 2015, Korean Society for Parasitology and Tropical Medicine

This is an Open Access article distributed under the terms of the Creative Commons Attribution Non-Commercial License (http://creativecommons.org/licenses/by-nc/3.0) which permits unrestricted non-commercial use, distribution, and reproduction in any

medium, provided the original work is properly cited. in transmitting the human immunodeficiency virus (HIV) type-1, and infection of women with T. vaginalis notably increases the risk of HIV infection [1,5].

In the female reproductive tract, the mucosal epithelial system is the first line of defense against pathogenic organisms [6-8]. Women with T. vaginalis infection in cervicovaginal mucosa secrete higher levels of proinflammatory cytokines such as IL- 6 , IL- $1 \beta$, and tumor necrosis factor- $\alpha$ (TNF- $\alpha$ ) [9]. The inflammatory response is regulated by various signaling pathways including mitogen-activated protein kinase (MAPK), phosphoinositide 3-kinase (PI3K), toll-like receptor (TLR), and nuclear factor- $\mathrm{\kappa B}$ (NF- $\kappa \mathrm{B})$ during the early stage of infection in host cells [10-12]. The PI3K pathways are a family of enzymes involved in cellular functions such as cell growth, proliferation, differentiation, motility, survival, and intracellular trafficking. They have been linked to an extraordinarily diverse group of cellular functions, and both directly and indirectly regulate the inflammatory pathway through the action of a serine/threonine kinase known as protein kinase $\mathrm{B}$ (PKB) 
or AKT, which plays an important role in the regulation of inflammatory responses as well as the survival of cells [10]. However, there are no reports of the role of the PI3K/AKT pathway in T. vaginalis-infected cervicovaginal mucosal epithelium.

MAPK signaling pathways are involved in a wide variety of cellular responses including inflammation, proliferation, differentiation, and apoptosis in all eukaryotic organisms. The MAPK forms a family of protein kinases that includes extracellular signal-regulated kinase 1/2 (ERK1/2), p38 MAPK, and cJun N-terminal kinase 1/2 (JNK1/2) [13]. There have been some investigations of proinflammatory cytokine production in various cells infected with T. vaginalis [9,14-17]; however, the roles of the PI3K/AKT and MAPK signaling pathways for TNF- $\alpha$ production in T. vaginalis-infected cervical mucosal epithelial cells remain to be elucidated.

Several studies have indicated that T. vaginalis infection increases proinflammatory responses in in vitro models $[9,14-$ 17]. TNF- $\alpha$ is a multifunctional cytokine that transduces signals for survival, differentiation, and cell death in diverse cell types and evokes diverse biological processes by inducing expression of various genes [18]. TNF- $\alpha$ plays an important role in the inflammatory response to trichomoniasis [14-16]. However, there is little information about the signaling pathways of TNF- $\alpha$ production in T. vaginalis-infected cervical mucosal epithelial cells. To elucidate the roles of the PI3K/AKT and MAPK pathways for TNF- $\alpha$ production in the cervical mucosal epithelium after T. vaginalis infection, human cervical mucosal epithelium SiHa cells were infected with $T$. vaginalis, and TNF- $\alpha$ production and phosphorylation of PI3K/AKT and MAPK pathways were evaluated in the presence and absence of specific inhibitors using western blotting or ELISA.

\section{MATERIALS AND METHODS}

\section{Antibodies and reagents}

The rabbit monoclonal anti-phospho ERK1/2 (p-ERK1/2), rabbit polyclonal anti-ERK1/2 (ERK1/2), rabbit monoclonal anti-phospho p38 MAPK (p-p38 MAPK), rabbit polyclonal anti-p38 MAPK (p38 MAPK), rabbit monoclonal anti-phospho JNK1/2 (p-JNK1/2), rabbit polyclonal anti-JNK1/2 (JNK1/2), rabbit polyclonal anti-phospho AKT (p-AKT), rabbit polyclonal anti-AKT (AKT) (all from Cell Signaling Technology, Beverly, Massachusetts, USA), mouse monoclonal anti-a tubulin (Santa Cruz Biotechnology, Santa Cruz, California, USA) were purchased from commercial companies. PI3K inhibitor wortman- nin and horseradish peroxidase-conjugated anti-rabbit IgG were purchased from Santa Cruz Biotechnology. PD98059 (2'-amino-3'-methoxyflavone; Calbiochem, San Diego, California, USA), which is a non-competitive inhibitor that is specific for the ERK1/2 family, prevents MEK1/2 activation by Raf. SB203580 [4-(4-fluorophenyl)-2-(4-methylsulfinylphenyl)5-(4-pyridyl) 1 H-imidazole; Calbiochem] directly inhibits the activation of p38 MAPK, and SP600125 (1,9-Pyrazoloanthrone; Calbiochem) preferentially inhibits JNK1/2.

\section{T. vaginalis culture}

The T016 strain of T. vaginalis [19] was kindly provided by Prof. Jae-Sook Ryu (Hanyang University College of Medicine, Seoul, Korea), and cultured in a glass, screw-capped tube containing Diamond's trypticase-yeast extract-maltose (TYM) medium (NAPCO, Winchester, Virginia, USA) supplemented with 10\% heat-inactivated horse serum (Sigma-Aldrich, St. Louis, Missouri, USA) in $5 \% \mathrm{CO}_{2}$ at $37^{\circ} \mathrm{C}$ for $24 \mathrm{hr}$. Cultured parasites were monitored for motility, and the viability of $T$. vaginalis was determined before each experiment using trypan blue staining (>99\%).

\section{Culture of SiHa cells}

The human cervical mucosal epithelial cancer cell line, SiHa, was obtained from the American Type Culture Collection (ATCC, Manassas, Virginia, USA) and maintained in Dulbecco's Modified Eagle's Medium (DMEM) supplemented with 10\% heat-inactivated fetal bovine serum (FBS; Gibco BRL, Grand Island, New York, USA) and antibiotic-antimycotic (Gibco BRL) in a $5 \% \mathrm{CO}_{2}$ atmosphere at $37^{\circ} \mathrm{C}$.

In a preliminary experiment to determine the adequate $T$. vaginalis/SiHa cell ratio that induces TNF- $\alpha$ production, $\mathrm{SiHa}$ cell monolayers $\left(1 \times 10^{6}\right)$ were washed with PBS ( $\left.\mathrm{pH} 7.4\right)$, and live T. vaginalis trophozoites were incubated in mixed-medium (DMEM/TYM = 2:1) at multiplicities of infection (MOIs) of $0.5,1,2$, and 5 for $2 \mathrm{hr}$ or T. vaginalis MOI 2 for $0.5,1,2,4$, and $8 \mathrm{hr}$. From these preliminary tests, MOI 2 of live T. vaginalis and $2 \mathrm{hr}$ incubation were selected as the adequate parasite number and incubation time, respectively, of live T. vaginalis to produce TNF- $\alpha$ in SiHa cells.

\section{Study design}

SiHa cells were seeded on 24-well plates (for ELISA assay) or $100 \mathrm{~mm}$ culture dishes (for western blotting) at various densities, and grown to confluence at $37^{\circ} \mathrm{C}$ in $5 \% \mathrm{CO}_{2}$. The profiles 
of TNF- $\alpha$ production and phosphorylation of PI3K/AKT and MAPK pathways in T. vaginalis-infected SiHa cells were evaluated by ELISA and western blot according to parasite incubation time, respectively. Before T. vaginalis infection to SiHa cells, cells were rested with FBS for $6 \mathrm{hr}$. To investigate the effects of PI3K/AKT and MAPK signaling pathways for production of TNF- $\alpha$, T. vaginalis-infected SiHa cells were pretreated with PI3K or MAPK inhibitors and evaluated TNF- $\alpha$ production in the culture supernatants later. The control was SiHa cells that were not infected with T. vaginalis. Each experiment was performed at least in triplicate.

\section{Cell viability by MTT}

SiHa cell viability after treatment with inhibitors was determined using MTT assay. Briefly, cells were seeded at a density of $5 \times 10^{3}$ cells/well in 96-well plates. The plates were incubated at $37^{\circ} \mathrm{C}$ for $24 \mathrm{hr}$ for adhesion of SiHa cells to the bottom of the well. Different concentrations of inhibitors were added to each well of 96-well plates for $24 \mathrm{hr}$. Then, $10 \mu \mathrm{l}$ of MTT solution $(5 \mathrm{mg} / \mathrm{ml})$ was added, and cells were further incubated at $37^{\circ} \mathrm{C}$ for $4 \mathrm{hr}$. Following the removal of MTT solution, waterinsoluble formazan was dissolved by adding $100 \mu \mathrm{l}$ dimethyl sulfoxide (DMSO) to each well. Finally, optical density (OD) was measured at $570 \mathrm{~nm}$ using microplate reader (Sunnyvale, California, USA). Data are shown as the absorbance of the untreated control.

\section{Western blotting}

SiHa cells were treated as indicated conditions and cells were washed in PBS, suspended in $5 \times$ SDS sample buffer and then heated at $98^{\circ} \mathrm{C}$ for $5 \mathrm{~min}$. The detergent-insoluble material was pelleted by centrifugation at 15,000 rpm for $5 \mathrm{~min}$ at $4^{\circ} \mathrm{C}$. The supernatants containing protein cell lysate were used for western blotting assays. Briefly, equal amounts of protein from each sample were separated by SDS-PAGE and transferred to a polyvinylidene difluoride membrane. The membranes were blocked in Tris-buffered saline (20 mM Tris, 137 $\mathrm{mM} \mathrm{NaCl}, \mathrm{pH} 7.6$ ) containing $0.1 \%$ Tween-20 (TBST) and 5\% skim milk. After a wash in TBST, membranes were incubated overnight at $4^{\circ} \mathrm{C}$ with the primary antibodies diluted in TBST supplemented with $5 \%$ BSA. The antibodies used were: anti-pAKT, anti-AKT, anti-p-ERK1/2, anti-ERK1/2, anti-p-p38 MAPK, anti-p38 MAPK, anti-p-JNK1/2, anti-JNK1/2, and anti- $\alpha$ tubulin. Following 3 consecutive washes in TBST, membranes were incubated for $90 \mathrm{~min}$ with horseradish peroxidase-conjugated anti-mouse or anti-rabbit IgG (Santa Cruz Biotechnology) diluted 1:10,000 with incubation buffer, as described above. After extensive washing, bound secondary antibodies were visualized using an enhanced ECL chemiluminescence detection kit (GE Healthcare, Little Chalfont, UK).

\section{Detection of TNF-a production by ELISA}

SiHa cells $\left(1 \times 10^{5}\right)$ were cultured in standard 12-well plates and then treated with medium or T. vaginalis MOI 2 for 1,2 , and $3 \mathrm{hr}$ in the presence or absence of PI3K inhibitor wortmannin (2, 20, or $200 \mathrm{nM})$, ERK1/2 inhibitor PD98059 (2, 10, or $50 \mu \mathrm{M})$, p38 MAPK inhibitor SB203580 $(1,5$, or 25 $\mu \mathrm{M})$ or JNK1/2 inhibitor SP600125 $(2,10$, or $50 \mu \mathrm{M})$. Culture supernatants were collected and stored at $-70^{\circ} \mathrm{C}$. TNF- $\alpha$ production from T. vaginalis-infected SiHa cells were analyzed with ELISA kits purchased from R\&D Systems (Minneapolis, Minneapolis, USA). All assays were performed in accordance with manufacturer's specifications.

\section{Statistical analysis}

The data are presented as means \pm SD. Statistical significance was determined by ANOVA (StatView; Abacus Concepts Inc., Berkeley, California, USA). All experiments were performed in at least triplicate on separate days. Differences were considered significant at $P$-values $<0.05$.

\section{RESULTS}

\section{T. vaginalis induced secretion of TNF-a in SiHa cells}

Proinflammatory cytokines were reported to be induced in various cell lines, including HeLa cells and macrophages, by $T$. vaginalis infection [15-17]. To investigate whether T. vaginalis infection induced TNF- $\alpha$ production in cervical mucosal epithelium, TNF- $\alpha$ levels in T. vaginalis-infected SiHa cells were measured from culture supernatants by ELISA according to parasite load and incubation time.

TNF- $\alpha$ production was significantly increased from MOI 0.5 of T. vaginalis and peaked at MOI 1 or 2 of T. vaginalis. However, T. vaginalis MOI 10-infected SiHa cells showed the basal levels of TNF- $\alpha$ (Fig. 1A). Thus, T. vaginalis MOI 2 was selected as a suitable parasite burden to induce TNF- $\alpha$ from SiHa cells. TNF- $\alpha$ production was also evaluated according to incubation times at T. vaginalis MOI 2. The amount of TNF- $\alpha$ production was significantly increased from 30 min post-infection $(P<0.01)$, and peaked at $4 \mathrm{hr}$ after infection $(P<0.001)$; thereafter, TNF- $\alpha$ 


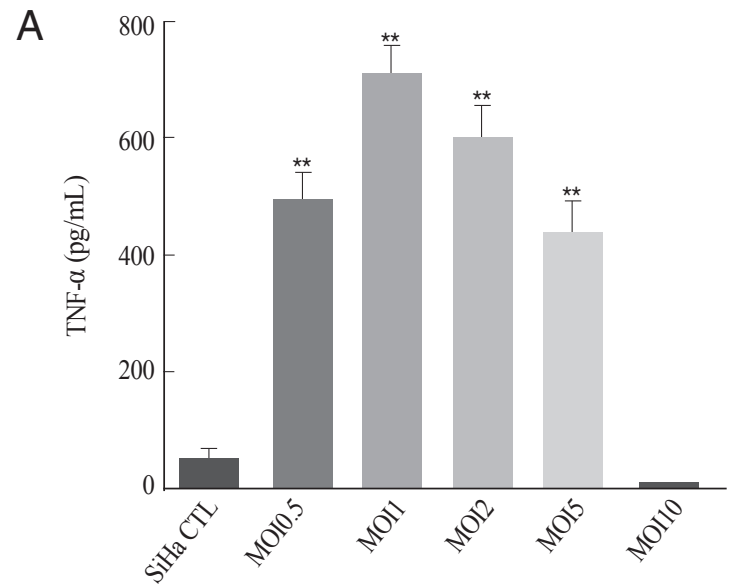

B

Fig. 1. T. vaginalis increased the secretion of the proinflammatory cytokine TNF- $\alpha$ in SiHa cells. SiHa cells were infected with T. vaginalis at a multiplicity of infection (MOI) 0.5, 1, 2, 5, and $10 \mathrm{for} 2 \mathrm{hr}(\mathrm{A})$ and infected with $T$. vaginalis $\mathrm{MOI} 2$ for $0.5,1,2,4,8$, and 16 hr (B), and then the levels of TNF-a (pg/ml) were analyzed in the culture supernatants by ELISA. The experiment was repeated 3 times with similar results. Data are presented as the means $\pm \mathrm{SD}$. ${ }^{* \star} P<0.05$ compared to the control (Ctl).

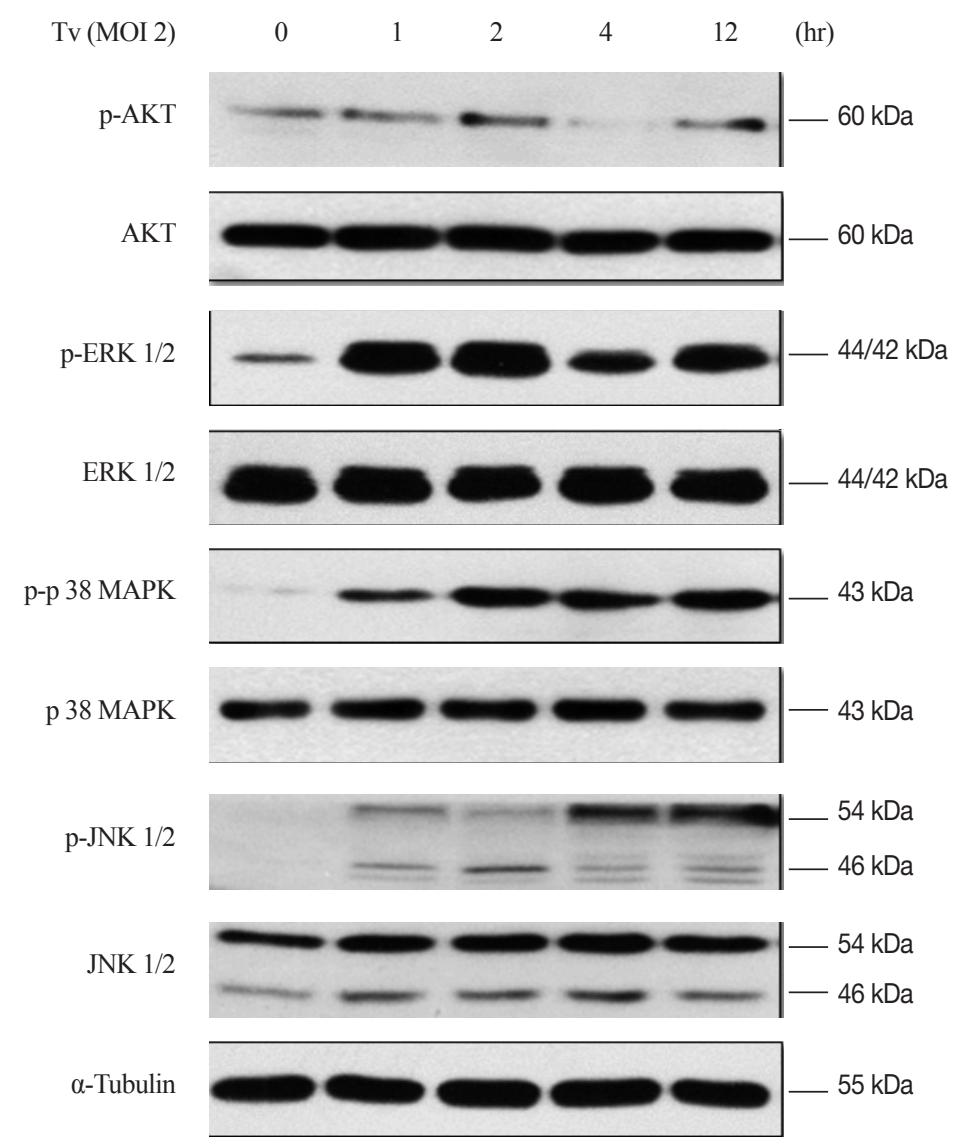

Fig. 2. T. vaginalis infection phosphorylated PI3K/KKT and MAPK pathways in SiHa cells. SiHa cells were treated with live T. vaginalis at $\mathrm{MOI} 2$, and incubated at $37^{\circ} \mathrm{C}, 5 \% \mathrm{CO}_{2}$ for $0.5,1,2$, and $4 \mathrm{hr}$. The cell pellets were harvested and analyzed by western blotting using antibodies to p-AKT, p-ERK1/2, p-p38 MAPK, p-JNK1/2, AKT, ERK1/2, p38 MAPK, JNK1/2, and a-tubulin. Similar results were obtained in 3 independent experiments. 
levels decreased (Fig. 1B). Thus, T. vaginalis MOI 2 for $2 \mathrm{hr}$ was selected as an adequate condition for the production of TNF- $\alpha$ in SiHa cells. The levels of TNF- $\alpha$ production at T. vaginalis MOI 2 for $2 \mathrm{hr}$ were 11-fold higher than that of the control $(610 \pm 55$ $\mathrm{pg} / \mathrm{ml}$ at 4 hr vs. $52 \pm 8 \mathrm{pg} / \mathrm{ml}$ at control; $P<0.001)$

\section{T. vaginalis infection induced activation of AKT, ERK1/2, p38 MAPK, and JNK1/2 in SiHa cells}

We investigated whether live T. vaginalis induced phosphorylation of AKT (Ser473) and MAPK signaling pathways in SiHa cells by western blot analysis. The phosphorylation of AKT was

A
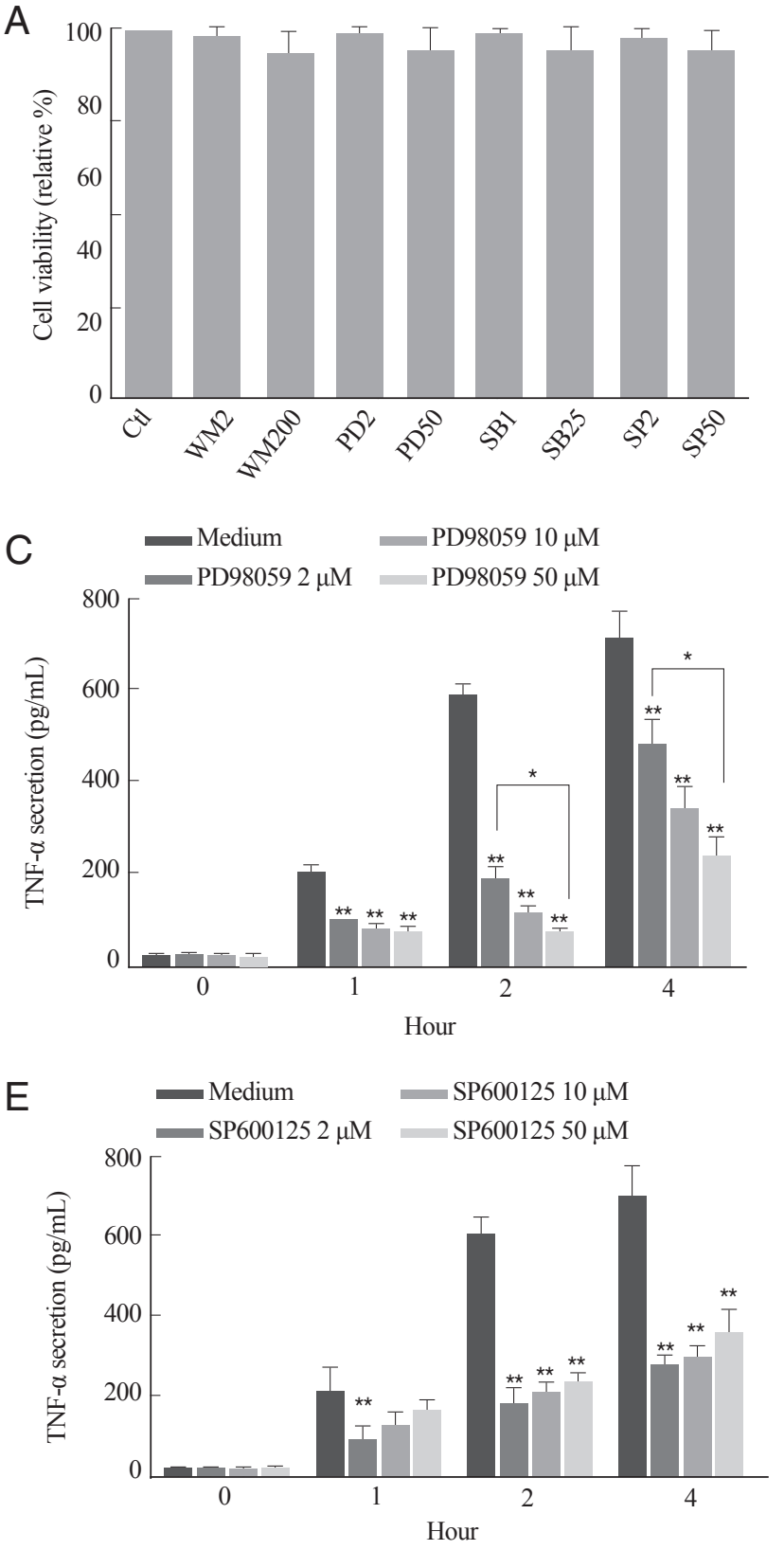

enhanced from $1 \mathrm{hr}$ after T. vaginalis infection (MOI 2) compared to the untreated control cells, peaked at $2 \mathrm{hr}$ and then decreased. However, there were no significant differences in the levels of unphosphorylated AKT compared to the control group (Fig. 2). The levels of p-ERK1/2, p-p38 MAPK, and p-JNK1/2 in T. vaginalis-infected SiHa cells were increased from $1 \mathrm{hr}$ after the infection, even though their activation kinetics were different each other. The phosphorylation levels of ERK1/2 were peaked at 1-2 hr after infection, and p-p38 MAPK levels were similar during 2-12 hr. However, p-JNK1/2 levels kept enhanced in the presence of the parasites until $12 \mathrm{hr}$. The protein levels of un-
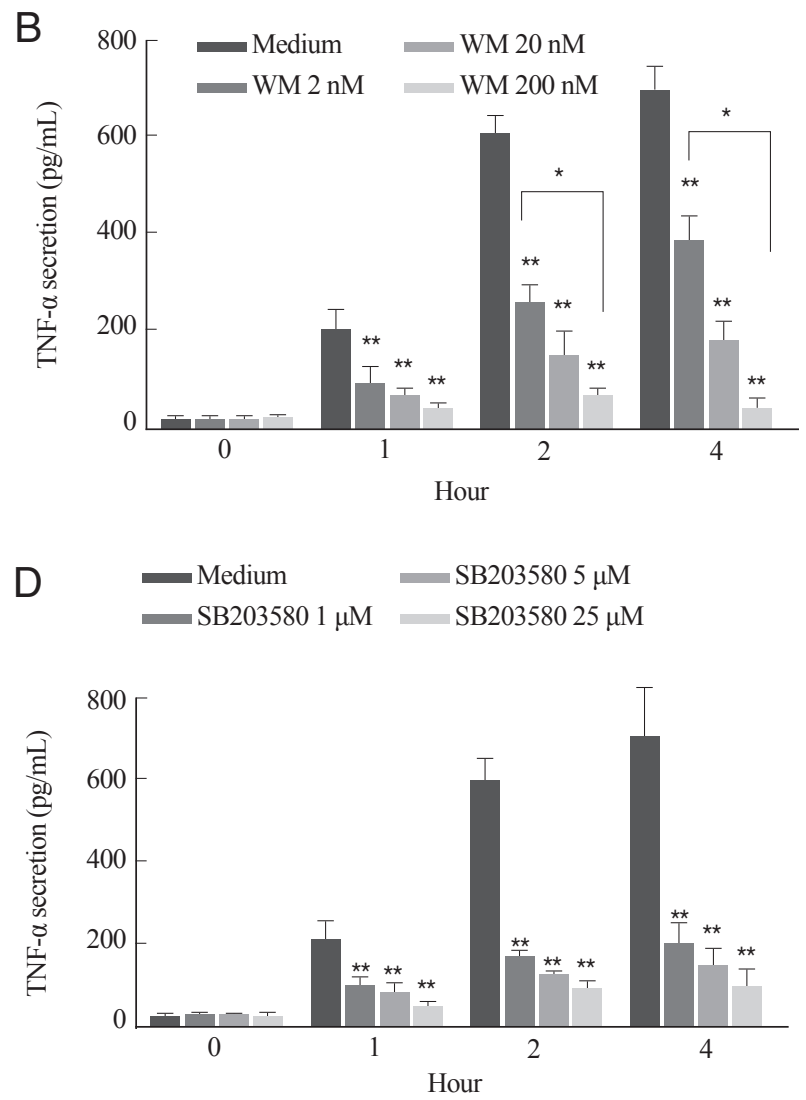

Fig. 3. Effects of PI3K and MAPK inhibitors at TNF-a production in T. vaginalis-infected SiHa cells. (A) Viability of SiHa cells after treatment with inhibitors of PI3K and MAPK pathways. (B-E) SiHa cells were pretreated with 2, 20, and $200 \mathrm{nM}$ of PI3K inhibitor wortmannin (B), 2, 10, and $50 \mu \mathrm{M}$ of ERK1/2 inhibitor PD98059 (C), 1, 5, and $25 \mu \mathrm{M}$ of p38 MAPK inhibitor SB203580 (D), and 2, 10 , and $50 \mu \mathrm{M}$ of JNK1/2 inhibitor SP600125 (E) for $1 \mathrm{hr}$, and treated with live T. vaginalis $\mathrm{MOI} 2$ for 1,2 , and $4 \mathrm{hr}$. The levels of TNF-a $(\mathrm{pg} / \mathrm{ml})$ in the culture supernatants were analyzed by ELI$\mathrm{SA}$. Data are presented as the means $\pm \mathrm{SD}$. *Statistically significant difference between 2 groups. ${ }^{\star \star} P<0.05$ compared to the medium-treated control. The experiment was repeated 3 times with similar results. 
phosphorylated AKT, ERK1/2, p38 MAPK, and JNK1/2 levels in the cells were similar to the control group (Fig. 2).

\section{TNF-a production in T. vaginalis-infected SiHa cells was significantly inhibited after treatment with inhibitors of PI3K/MAPK pathways}

To investigate whether the PI3K/AKT and MAPK pathways are involved in the TNF- $\alpha$ secretion from T. vaginalis-infectedSiHa cells, the levels of TNF- $\alpha$ production were evaluated in SiHa cells pretreated with various types of specific inhibitors. The cytotoxic effects of these inhibitors against SiHa cells were evaluated by MTT assay. There was no significant cellular toxicity even in the pretreatment of $200 \mu \mathrm{M}$ wortmannin, $50 \mu \mathrm{M}$ PD98059, 25 M SB203580, or $50 \mu \mathrm{M}$ SP600125 (Fig. 3A).

TNF- $\alpha$ production was gradually increased until $4 \mathrm{hr}$ in $T$. vaginalis-infected SiHa cells in a time-dependent manner (Fig. 1B). After pretreatment with the PI3K or MAPKs inhibitors, TNF- $\alpha$ production significantly decreased in comparison to medium-treated control group at each time point $(P<0.001)$; however, the inhibitory pattern of each inhibitor was different each other (Fig. 3B-E). In detail description of the kinetics of TNF- $\alpha$ reduction, TNF- $\alpha$ productions were decreased in a dosedependent manner at each time point after pretreatment with PI3K inhibitor wortmannin $(P<0.05$, Fig. 3B), ERK1/2 inhibitor PD98059 $(P<0.05$, Fig. 3C), and p38 MAPK inhibitor SB203580 ( $P>0.05$, Fig. 3D). In contrast, pretreatment of JNK1/2 inhibitor SP600125 increased the TNF-a production with increment of inhibitor $(P>0.05$, Fig. 3E). These data suggest that the PI3K/AKT and MAPK pathways are involved in the TNF- $\alpha$ secretion from T. vaginalis-infected SiHa cells; however, their activation kinetics were different from each pathway.

\section{DISCUSSION}

T. vaginalis evokes inflammatory responses during epithelial cell infection [7,14], and TNF- $\alpha$ plays an important role in the inflammatory response to trichomoniasis [9,14-17]. In this study, TNF-a production increased in SiHa cells infected with $T$. vaginalis in a parasite dose- and incubation time-dependent manner. Interestingly, TNF-a production significantly increased 30 min after T. vaginalis infection in SiHa cells, peaked $4 \mathrm{hr}$ postinfection, decreased thereafter, and virtually returned to its basal at $16 \mathrm{hr}$. These results were similar to previous reports that TNF- $\alpha$ production significantly increased from $1 \mathrm{hr}$ in $T$. vaginalis-infected macrophages and dramatically decreased at 8 hr due to inhibition of nuclear translocation of NF-kB [16]. In addition, TNF- $\alpha$ levels were almost at basal levels in T. vaginalis MOI 10 after $2 \mathrm{hr}$ incubation. These data meant that a high number of parasites may produce higher levels of proteinases and induce intensive cell death $[19,20]$.

The PI3K/AKT and MAPK signaling pathways are mediated by several groups of sequentially activated protein kinases [10]. In this study, T. vaginalis infection induced the phosphorylation of p-AKT, p-ERK1/2, p-p38 MAPK, and p-JNK1/2 from 1 $\mathrm{hr}$ after infection in SiHa cells. These results were partly consistent with Ryang et al. [21], who showed that T. vaginalis-infected macrophages induced the phosphorylation of ERK1/2, p38 MAPK, and JNK1/2 at 40 min after infection. Interestingly, the activation kinetics of phosphorylated AKT, ERK1/2, p38 MAPK, and JNK1/2 were different from each other. The levels of p-AKT and p-ERK1/2 were peaked within $2 \mathrm{hr}$ after T. vaginalis infection and then decreased; however, p-JNK1/2 levels were peaked at $12 \mathrm{hr}$ postinfection. These data suggest that $T$. vaginalis infection induced the activation of PI3K and MAPK pathways in SiHa cells; however, the activation patterns were different from the types of PI3K/MAPK pathways.

Next, we evaluated the roles of PI3K/AKT and MAPK pathways in TNF- $\alpha$ production using specific inhibitors. Our data showed that TNF- $\alpha$ productions were significantly decreased after pretreatment with inhibitors of PI3K and MAPK pathways in T. vaginalis-infected SiHa cells compared to the control group. These data demonstrated that TNF- $\alpha$ production was regulated by PI3K/AKT and MAPK pathways in cervical mucosal epithelium after T. vaginalis infection. Similar results were also indicated by some authors that TNF- $\alpha$ production was caused by activation of PI3K/AKT and MAPK pathways $[15,16,21,22]$. However, the detailed patterns of TNF- $\alpha$ reduction at each time point were different from inhibitors of the PI3K/AKT and MAPK pathways. These data suggested that PI3K/AKT and MAPK pathways are important in TNF- $\alpha$ production in T. vaginalis-infected cervical mucosal epithelial SiHa cells, although the action mode of each pathway was different. Although PI3K/AKT and MAPK pathways are important in TNF- $\alpha$ production, TNF- $\alpha$ production was positively regulated by the PI3K/AKT, ERK/12, and p38 MAPK pathways in T. vaginalis-infected SiHa cells, whereas JNK1/2 pathway regulated TNF- $\alpha$ production negatively. Similar results were presented at the previous reports that ERK, p38 MAPK, and NF- $\mathrm{kB}$ pathways play important roles in the inflammatory responses at $T$. vaginalis-infected HeLa cells [15], and T. vaginalis-induced regu- 
lation of TNF- $\alpha$ gene expression is at least partially dependent on p38 MAPK in HeLa cells [16]. It was also reported that negative regulation of JNK activation may play a critical role in cell survival in response to TNF- $\alpha$ in human breast carcinoma MCF-7 and mouse fibroblast LM cells [23].

Collectively, our data suggested that PI3K/AKT and MAPK signaling pathways are important in regulation of TNF- $\alpha$ production in cervical mucosal epithelial SiHa cells, although the activation pattern of each pathway was different from the types of PI3K/MAPK pathways.

\section{ACKNOWLEDGMENT}

This work was supported by a research fund of Chungnam National University, 2014.

\section{CONFLICT OF INTEREST}

There are no conflicts of interest for any of the authors.

\section{REFERENCES}

1. Johnston VJ, Mabey DC. Global epidemiology and control of Trichomonas vaginalis. Curr Opin Infect Dis. 2008; 21: 56-64.

2. Ryan CM, de Miguel N, Johnson PJ. Trichomonas vaginalis: current understanding of host-parasite interactions. Essays Biochem 2011; 51: 161-175.

3. Van der Pol B. Trichomonas vaginalis infection: the most prevalent nonviral sexually transmitted infection receives the least public health attention. Clin Infect Dis 2007; 44: 23-25.

4. Coleman JS, Gaydos CA, Witter F. Trichomonas vaginalis vaginitis in obstetrics and gynecology practice: new concepts and controversies. Obstet Gynecol Surv 2013; 68: 43-50.

5. Guenthner PC, Secor WE, Dezzutti CS. Trichomonas vaginalis-induced epithelial monolayer disruption and human immunodeficiency virus type 1 (HIV-1) replication: implications for the sexual transmission of HIV-1. Infect Immun 2005; 73: 41554160.

6. Quayle AJ. The innate and early immune response to pathogen challenge in the female genital tract and the pivotal role of epithelial cells. J Reprod Immunol 2002; 57: 61-79.

7. Wira CR, Grant-Tschudy KS, Crane-Godreau MA. Epithelial cells in the female reproductive tract: a central role as sentinels of immune protection. Am J Reprod Immunol 2005; 53: 65-76.

8. Cole AM. Innate host defense of human vaginal and cervical mucosae. Curr Top Microbiol Immunol 2006; 306: 199-230.

9. Malla N, Yadav M, Gupta I. Kinetics of serum and local cytokine profile in experimental intravaginal trichomoniasis induced with Trichomonas vaginalis isolates from symptomatic and as- ymptomatic women. Parasite Immunol 2007; 29: 101-105.

10. Aksamitiene E, Kiyatkin A, Kholodenko BN. Cross-talk between mitogenic Ras/MAPK and survival PI3K/Akt pathways: a fine balance. Biochem Soc Trans 2012; 40: 139-146.

11. Yang ZZ, Tschopp O, Baudry A, Dummler B, Hynx D, Hemmings BA. Physiological functions of protein kinase B/Akt. Biochem Soc Trans 2004; 32: 350-354.

12. Gasparini C, Feldmann M. NF- $\mathrm{KB}$ as a target for modulating inflammatory responses. Curr Pharm 2012; 18: 5735-5745.

13. Cargnello M, Roux PP. Activation and function of the MAPKs and their substrates, the MAPK-activated protein kinases. Microbiol Mol Biol Rev 2011; 75: 50-83.

14. Paintlia MK, Kaur S, Gupta I, Ganguly NK, Mahajan RC, Malla N. Specific IgA response, T-cell subtype and cytokine profile in experimental intravaginal trichomoniasis. Parasitol Res 2002; 88: 338-343.

15. Lin MC, Hui CF, Chen JY, Wu JL. The antimicrobial peptide, shrimp anti-lipopolysaccharide factor (SALF), inhibits proinflammatory cytokine expressions through the MAPK and NF-kB pathways in Trichomonas vaginalis adherent to HeLa cells. Peptides 2012; 38: 197-207.

16. Chang JH, Ryang YS, Morio T, Lee SK, Chang EJ. Trichomonas vaginalis inhibits proinflammatory cytokine production in macrophages by suppressing NF-kB activation. Mol Cells 2004; 18 : 177-185.

17. Han IH, Goo SY, Park SJ, Hwang SJ, Kim YS, Yang MS, Ahn MH, Ryu JS. Proinflammatory cytokine and nitric oxide production by human macrophages stimulated with Trichomonas vaginalis. Korean J Parasitol 2009; 47: 205-212.

18. Aggarwal BB, Shishodia S, Ashikawa K, Bharti AC. The role of TNF and its family members in inflammation and cancer: lessons from gene deletion. Curr Drug Targets Inflamm Allergy 2002; 1: 327-341.

19. Quan JH, Kang BH, Cha GH, Zhou W, Koh YB, Yang JB, Yoo HJ, Lee MA, Ryu JS, Noh HT, Kwon J, Lee YH. Trichonomas vaginalis metalloproteinase induces apoptosis of SiHa cells through disrupting the $\mathrm{Mcl}-1 / \mathrm{Bim}$ and $\mathrm{Bcl}-\mathrm{xL} / \mathrm{Bim}$ complexes. PLoS One 2014; 9: e110659.

20. Quan JH, Choi IW, Yang JB, Zhou W, Cha GH, Zhou Y, Ryu JS, Lee YH. Trichomonas vaginalis metalloproteinase induces mTOR cleavage of SiHa cells. Korean J Parasitol 2014; 52: 595-603.

21. Ryang YS, Chang JH, Park JY. Involvement of MAP kinases in apoptosis of macrophage treated with Trichomonas vaginalis. Yonsei Med J 2004; 45: 751-754.

22. Zhou Z, Gengaro P, Wang W, Wang XQ, Li C, Faubel S, Rivard C, Schrier RW. Role of NF-kappaB and PI 3-kinase/Akt in TNF-alpha-induced cytotoxicity in microvascular endothelial cells. Am J Physiol Renal Physiol 2008; 295: F932-F941.

23. Tang F, Tang G, Xiang J, Dai Q, Rosner MR, Lin A. The absence of NF-kB-mediated inhibition of c-Jun N-terminal kinase activation contributes to tumor necrosis factor alpha-induced apoptosis. Mol Cell Biol 2002; 22: 8571-8579. 
